D.O.I: $10.3895 / \mathrm{S} 1808-04482013000300009$

\title{
MODULARIDADE E DESDOBRAMENTO DA FUNÇÃO QUALIDADE: UMA ANÁLISE TEÓRICA DE PUBLICAÇÕES
}

\section{MODULARITY AND QUALITY FUNCTION DEPLOYMENT: A THEORETICAL ANALYSIS OF PUBLICATIONS}

\author{
Flávio Issao Kubota ${ }^{1}$; Paulo Augusto Cauchick Miguel $^{2}$ \\ ${ }^{1}$ Universidade Federal de Santa Catarina - UFSC - Florianópolis - Brasil \\ flavioissao.kubota@gmail.com \\ ${ }^{2}$ Universidade Federal de Santa Catarina - UFSC - Florianópolis - Brasil \\ cauchick@deps.ufsc.br
}

\begin{abstract}
Resumo
As organizações, crescentemente, têm sido pressionadas a produzirem bens e serviços de maior qualidade e adequação aos clientes e consumidores. Junto a isso, a pressão quanto à redução do tempo de desenvolvimento de produtos e aumento de variedade dos mesmos também é cada vez. mais intensa. Assim, o presente trabalho tem como objetivo identificar e analisar publicações sobre a aplicação integrada da modularidade ao desdobramento da função qualidade (QFD), que são conceitos amplamente utilizados nos últimos anos e considerados importantes na busca por maior qualidade e diversificação de produtos. Realizou-se uma pesquisa teórico-conceitual estruturada por meio de busca e análise da literatura acerca do assunto. Os resultados obtidos apresentam lacunas e oportunidades de pesquisa no tema, bem como características metodológicas e os resultados obtidos pelos artigos analisados. Assim, o trabalho evidencia, principalmente, que a maioria dos trabalhos explora apenas a primeira matriz do QFD e se utiliza de modelagem teórica como método de pesquisa, além de haver uma tendência recente de adotar os dois conceitos, integrados, no setor de serviços.
\end{abstract}

Palavras-chave: desdobramento da função qualidade; QFD; modularidade; análise teóricoconceitual.

\section{Introdução}

As organizações que não estão centradas na produção de qualidade e melhoria de posicionamento no mercado, dificilmente sobrevivem em um ambiente que se mostra cada vez mais exigente quanto à diferenciais competitivos de mercado e atendimento às necessidades e expectativas dos consumidores. O rápido avanço da tecnologia e economia criou uma intensa concorrência no mercado consumidor atual. As exigências dos consumidores de hoje em relação aos produtos estão em constante mudança e produtos que se mantém estagnados não satisfazem mais os desejos dos consumidores. A fim de captar novas oportunidades de negócios, as empresas têm sido 
pressionadas a fabricar diversos produtos para satisfazer as necessidades dos consumidores (KO; KUO, 2010).

Nesse contexto, torna-se necessário que as empresas desenvolvam produtos e serviços que conquistem a preferência dos clientes. Um dos métodos que contribui no alcance desse objetivo é o Desdobramento da Função Qualidade (QFD - Quality Function Deployment), que incrementa a aceitação do produto, e reduz o tempo de desenvolvimento do projeto (DEVADASAN et al, 2006), aumentando a satisfação dos clientes (LAGER, 2005). O QFD é um método de desenvolvimento de produto originado no Japão no início da década de 1960 (AKAO, 1990; CLAUSING, 1994; TERNINKO, 1997), que busca incorporar as demandas dos clientes ao longo do processo de projeto do produto, bem como no projeto dos sistemas de produção. Esse método converte a "voz do cliente" em termos de projeto, fabricação e produção para garantir que o produto atenda as necessidades dos clientes (WANG; LIN, 2007; PEROTTI et al, 2009; LIMA et al, 2012). Ainda, o QFD é uma técnica formal conhecida para desenvolvimento efetivo de produto, e é mais utilizada, normalmente, nas fases iniciais de projeto (HUNG et al, 2007).

Além do QFD, outra abordagem que tem sido amplamente utilizada pelas empresas é a modularidade. Esta abordagem tem sido considerada por trazer uma série de benefícios aos negócios das empresas, desde o desenvolvimento do produto até a produção, tais como economia de escala, aumento da viabilidade de mudanças, aumento na variedade de produtos, redução de leadtime, facilidade de projeto e testes e manutenção e diagnóstico de produtos mais facilitadas (PINE, 1993; KUSIAK; HUANG, 1996; PAHL; BEITZ, 1996; GERSHENSON et al, 2003; WANG, 2009; GEUM et al, 2012).

O conceito de modularidade surgiu na indústria de computadores durante a década de 1960, gerando vantagem competitiva e demonstrando significativa importância no processo de desenvolvimento de produto (ARNHEITER; HARREN, 2006). Este conceito visa a decomposição de produtos complexos em subsistemas que constituem unidades funcionais completas, as quais podem ser projetadas e produzidas de forma independente (o que permite a construção de diferentes produtos por meio da combinação de subsistemas) e com redução de complexidade, mas com seu funcionamento integrado (BALDWIN; CLARK, 1997; PERSSON, 2006). Nesse sentido, a modularidade apresenta-se como um conceito de significativa utilidade em diversas áreas que lidam com sistemas complexos (BALDWIN; CLARK, 2000), no setor automotivo (CARNEVALLI et al, 2011; PRIETO; CAUCHICK MIGUEL, 2011; CHRISTENSEN, 2011), de componentes eletrônicos (ARNHEITER; HARREN, 2006; HUANG et al, 2012), móveis (CARIDI et al, 2012) e serviços (LIN; PEKKARINEN, 2011; GEUM et al, 2012), entre outros, incluino a neurociência e psicologia até a robótica e inteligência artificial (BALDWIN; CLARK, 2000). 
Apesar de ser utilizado em diversos países, existem dificuldades na aplicação do uso do QFD, fato que vem limitando a sua utilização e dificultando a adoção do método por parte das empresas (CARNEVALLI et al, 2005). Nesse sentido, apenas o registro dos requisitos pode não ser o suficiente, pois as organizações, muitas vezes, não têm o aporte financeiro para atender igualmente a todas as necessidades. Assim, Qin e Wei (2012) destacam que se torna necessário classificá-las prioritariamente, transformando esses requisitos em informações atribuídas aos módulos de produto. Nesse sentido, considerando-se que a modularidade contribui no desenvolvimento de produtos por meio da redução de complexidade dos mesmos, acredita-se que a modularidade pode contribuir na diminuição das dificuldades relacionadas aos desdobramentos dos requisitos de cliente e as especificações de projeto. Nesse contexto, este trabalho tem como objetivo identificar e analisar as publicações acerca dos temas modularidade e QFD, com o intuito de investigar as características referentes aos principais resultados quanto aos tipos de abordagem, métodos de pesquisa adotados, níveis de aplicação do QFD, setores de maior utilização dos conceitos integrados, tipos de modularidade e métodos e técnicas adicionais utilizadas desses trabalhos, além da identificação de tendências e das principais oportunidades de pesquisas futuras no assunto.

Após esta introdução, o trabalho se encontra organizado conforme a seguinte sequência: na seção 2 é apresentada a revisão de literatura que fornece a estrutura teórica para o desenvolvimento do estudo e para a análise de conteúdo do portfólio de artigos. Em seguida, na seção 3, encontramse os procedimentos metodológicos usados para viabilização do trabalho. Por fim, nas seções 4 e 5 , respectivamente, são descritos os resultados encontrados e discussão dos mesmos e as conclusões e oportunidades para trabalhos futuros.

\section{Referencial teórico}

Esta fundaentação teórica envolve a base sobre os dois temas tratatos no presente trabalho: modularidade e desdobramento da função qualidade.

\subsection{Modularidade - conceitos e tipos de abordagem}

Os consumidores atuais necessitam de produtos personalizados, visando o atendimento mais preciso às suas necessidades. Esses consumidores anseiam por produtos que sejam únicos, ou seja, que expressem sua individualidade e os façam de destacar perante às demais pessoas. Assim, a variedade de produtos se tornou um dos aspectos mais importantes na conquista de novos segmentos de mercado, indicando que as empresas devem considerar o projeto de produtos variados como estratégia no desenvolvimento de produtos (KO; KUO, 2010). 
Dentro dessa perspectiva de oferecer variedade de produtos em conjunto com qualidade e menores custos de produção, encontram-se os princípios da modularidade. Para a aplicação da modularidade em projetos, diversas etapas e tomadas de decisões devem ser feitas, o que evidencia a complexidade dessa estratégia (ASAN et al, 2004). Assim como em outras estratégias e métodos, as decisões quanto ao grau de modularidade do produto e a escolha dos processos produtivos para a viabilização desses produtos afetam consideravelmente os custos do projeto. Inicialmente, dois tipos de modularidade se destacam: a primeira é a modularidade no projeto, que trata das fronteiras entre subsistemas de componentes que integrados formam o produto completo (MORRIS; DONNELLY, 2006). Essa abordagem visa a redução de lead-time e proporciona a concepção de módulos por fornecedores especializados em determinado módulo e/ou subsistema, o que gera contribuições em evolução tecnológica (GRAZIADIO, 2004). O segundo tipo, a modularidade na produção, surgiu como diferencial competitivo na década de 1960 e visa aperfeiçoar a montagem final do produto, permitindo o aumento de variedade sem o aumento de custos (CARNEVALLI et al, 2011).

No entanto, considerando-se as necessidades de maior personalização e produtos "únicos" desejados pelos consumidores atualmente, emergiu um terceiro tipo: a modularidade de uso, que é mais diretamente relacionada com o aumento de variedade e individualização de produtos, atendendo a usos diversos. Considerando o aumento de opções ofertadas, teoricamente é possível, desse modo, expandir as possibilidade de melhor atendimento aos anseios dos consumidores (CARDOSO; KISTMANN, 2008). Por meio dessa abordagem, a modularidade pode ser uma estratégia para obter diferenciação e customização de produtos, além de outras vantagens na perspectiva ambiental e de redução de custos. Ainda, Stäblein et al (2011) complementam afirmando que o compartilhamento de módulos, combinados em diferentes versões, viabilizam o aumento na variedade de produtos. No entanto, as empresas necessitam desenvolver uma arquitetura modular, que gere plataformas de produtos e, consequentemente, gerem produtos modulares (PELEGRINI, 2004).

Além desses três tipos de modularidade (projeto, produção e uso), recentemente, os conceitos de modularidade têm sido aplicados em outras duas perspectivas: a organizacional (CAMPAGNOLO; CAMUFFO, 2009; CHENG, 2011; TSVETSKOVA; GUSTAFSSON, 2012) e em serviços (LIN; PEKKARINEN, 2011; BASK et al, 2011; GEUM et al, 2012), com a possibilidade de desenvolver a modelagem de negócios por meio da modularidade (BASK et al, 2011). Cheng (2011) relata que a modularidade organizacional tem constituído uma potencial mudança de paradigma na gestão de sistemas complexos, sendo um fenômeno que continuará se desenvolvendo nos próximos anos. O autor citado afirma ainda que elevados níveis de modularidade organizacional estão associados a um alto nível de capacidade de utilização 
(recursos), retorno sobre o investimento (ROI) e retorno sobre o ativo (ROA), no entanto, também podem levar a um grau menor de especialização em produtos.

Em outro estudo, Tsvetskova e Gustafsson (2012), especificamente, propõem a redução de complexidade quanto ao gerenciamento de um eco-sistema industrial por meio da modularização funcional, visando resolver o problema de replicação de eco-sistemas industriais considerando não apenas as mudanças de materiais e recursos, mas também a questão empresarial. Ou seja, a modularidade organizacional tem por objetivo, semelhante ao caso de produtos, reduzir a complexidade no gerenciamento das atividades referentes ao negócio de uma empresa e/ou cadeia produtiva.

Por fim, a modularidade em serviços, ainda pouco explorada em um contexto prático, consiste em proporcionar uma análise sistemática dos serviços, decompondo o serviço principal para uma melhor avaliação (GEUM et al, 2012). Os mesmos autores argumentam que, por meio dessa abordagem, a modularidade tem potencial para desempenhar um papel crucial no setor de serviços. Esse conceito (modularidade em serviços) foi proposto, primeiramente, por Sundbo (1994), que apresentou a viabilidade e os benefícios dessa aplicação. No entanto, as dificuldades inerentes à modelagem de serviços mostra que poucos estudos foram feitos nessa perspectiva (PEKKARINEN; ULKUNIEMI, 2008).

\subsection{Desdobramento da função qualidade - QFD (Quality Function Deployment)}

Em uma época onde consumidores demandam por produtos customizados de alta qualidade e preço reduzido, a competição entre empresas tem abordado a variedade de produtos e a velocidade de resposta ao mercado, pois é necessário que se satisfaçam as exigências dos clientes na configuração do produto, no entanto (QIN; WEI, 2012). Para isso, é necessário que as empresas conheçam bem seus clientes por meio da identificação dos requisitos considerados mais importantes por esses, que devem ser atendidos de maneira suficiente pelos produtos oferecidos (CARNEVALLI; CAUCHICK MIGUEL, 2009).

O desdobramento da função qualidade (QFD), desse modo, é um importante método que contribui para o desenvolvimento de produtos que possam atender e superar as expectativas dos clientes (CARNEVALLI; CAUCHICK MIGUEL, 2009). Resumidamente, o QFD consiste em transformar as exigências dos clientes em características da qualidade, posteriormente transferindoas para as etapas seguintes do desenvolvimento de produtos, por meio de desdobramentos sucessivos (FORMAGGIO; CAUCHICK MIGUEL, 2009). Complementando, o QFD consiste de uma série de matrizes que calculam os valores das funções do produto, requisitos, componentes e requisitos de processo na importância dos requisitos de cliente (TAKAI; ISHII, 2006). 
A primeira matriz do QFD, usualmente chamada na literatura de "matriz - ou casa - da qualidade", é onde são estabelecidas a qualidade planejada e a qualidade projetada (FORMAGGIO; CAUCHICK MIGUEL, 2009). Trata-se de uma maneira gráfica de dispor as necessidades dos clientes, as métricas, o desempenho dos concorrentes e as especificações de metas (SPINELLA et al, 2009). Duas perspectivas são necessárias para definir a qualidade planejada: a perspectiva do cliente, considerando as características mais importantes para ele, e; a perspectiva da empresa, que se posiciona de forma comparativa com os concorrentes (AKAO, 1996). Após a definição da qualidade projetada para o produto, parte-se para o projeto do produto e do processo, visando atingir o objetivo geral do QFD por meio de outras matrizes de desdobramento. O sequenciamento dessas matrizes pode ser feito, por exemplo, para o desdobramento da tecnologia, desdobramento de custos e o desdobramento da confiabilidade (FORMAGGIO; CAUCHICK MIGUEL, 2009).

Esta fundamentação apresentou, então, uma base teórica a qual serviu como estrutura para a análise do portfólio bibliográfico de artigos sobre modularidade/QFD. As abordagens da modularidade, bem como o nível de aplicação do QFD nos estudos, foram investigados e analisados com o objetivo de avaliar as contribuições e aprofundamento nos conceitos alcançados nesses trabalhos e identificar oportunidades para futuros estudos. Antes da seção de resultados e discussões, são apresentados os procedimentos metodológicos utilizados para viabilizar o presente trabalho.

\section{Método de pesquisa}

O presente trabalho se caracteriza como teórico-conceitual (conforme FILIPPINI, 1997 e BERTO; NAKANO, 2000), pois visa realizar discussões conceituais a partir da literatura. Para fins de busca na literatura, as palavras-chave utilizadas nas bases de dados Scopus, ISI Web of Knowledge e Compendex foram as seguintes: "quality function deployment" e "QFD" combinadas com "modularity", "modularization", "modularisation", "modular", "modular product", "modular production", "modular service", "modular project" e "module" e combinações.

Na primeira base acessada (Scopus) foi utilizado filtro para remover áreas não relacionadas com o tema da pesquisa, tais como Bioquímica, Química Industrial, Ciências Sociais, Geologia, etc. Na segunda base (ISI Web of Knowledge) foi utilizado um filtro para remover artigos publicados em eventos ou conferências, ou seja, considerar apenas os trabalhos publicados em periódicos, assim como na terceira base (Compendex). Desse modo, apenas os artigos relacionados às áreas de administração, ciências contábeis, engenharias, e áreas correlatas foram considerados. Para a organização dos artigos e dos filtros de seleção, foi utilizado o software EndNote ${ }^{\circledR}$ X5 como suporte. 
Primeiramente, os artigos foram lidos nesta sequência: título, palavras-chave e resumo. Em seguida, se o artigo estava inserido no tema em questão (modularidade ou QFD), procedeu-se à leitura da introdução e conclusões dos artigos, seguidos da leitura completa dos mesmos. Nesta etapa, buscou-se avaliar quais artigos se encontravam alinhados ao escopo da pesquisa, removendose trabalhos considerados fora das áreas citadas. Posteriormente, foi possível realizar a análise de conteúdo dos trabalhos, desde a classificação científica das pesquisas e outros dados bibliométricos simples, até uma análise de conteúdo mais aprofundada, com o intuito de encontrar lacunas e oportunidades de pesquisa no estudo do QFD alinhado à modularidade, bem como verificar o panorama atual (estado da arte) das publicações.

$\mathrm{Na}$ análise de conteúdo, feita por meio do método hipotético-dedutivo (conforme NUNES; BENNET, 2008), foram observados os seguintes aspectos: abordagem de pesquisa (qualitativa ou quantitativa) e métodos de pesquisa (estudo de caso, modelagem teórica, survey, etc.), setores econômicos de aplicação, enfoque na aplicação do QFD (utilização restrita da $1^{\mathrm{a}}$ matriz de qualidade, uso de modelos conceituais com duas ou mais matrizes, etc.), relação do QFD com a modularidade, métodos e técnicas adicionais (além do QFD e modularidade), abordagem em produtos (bens de consumo) ou serviços e os tipos de modularidade aplicados (projeto, produção, uso, serviço e/ou organizacional). O propósito foi gerar um quadro teórico acerca das contribuições dos trabalhos desenvolvidos e tendências futuras para o tema. Além disso, um quadro de oportunidades para estudos futuros foi elaborado com base nas conclusões extraídas do portfólio de artigos. A Figura 1 resume as etapas e o sequenciamento do trabalho. 
Figura 1 - Procedimentos metodológicos

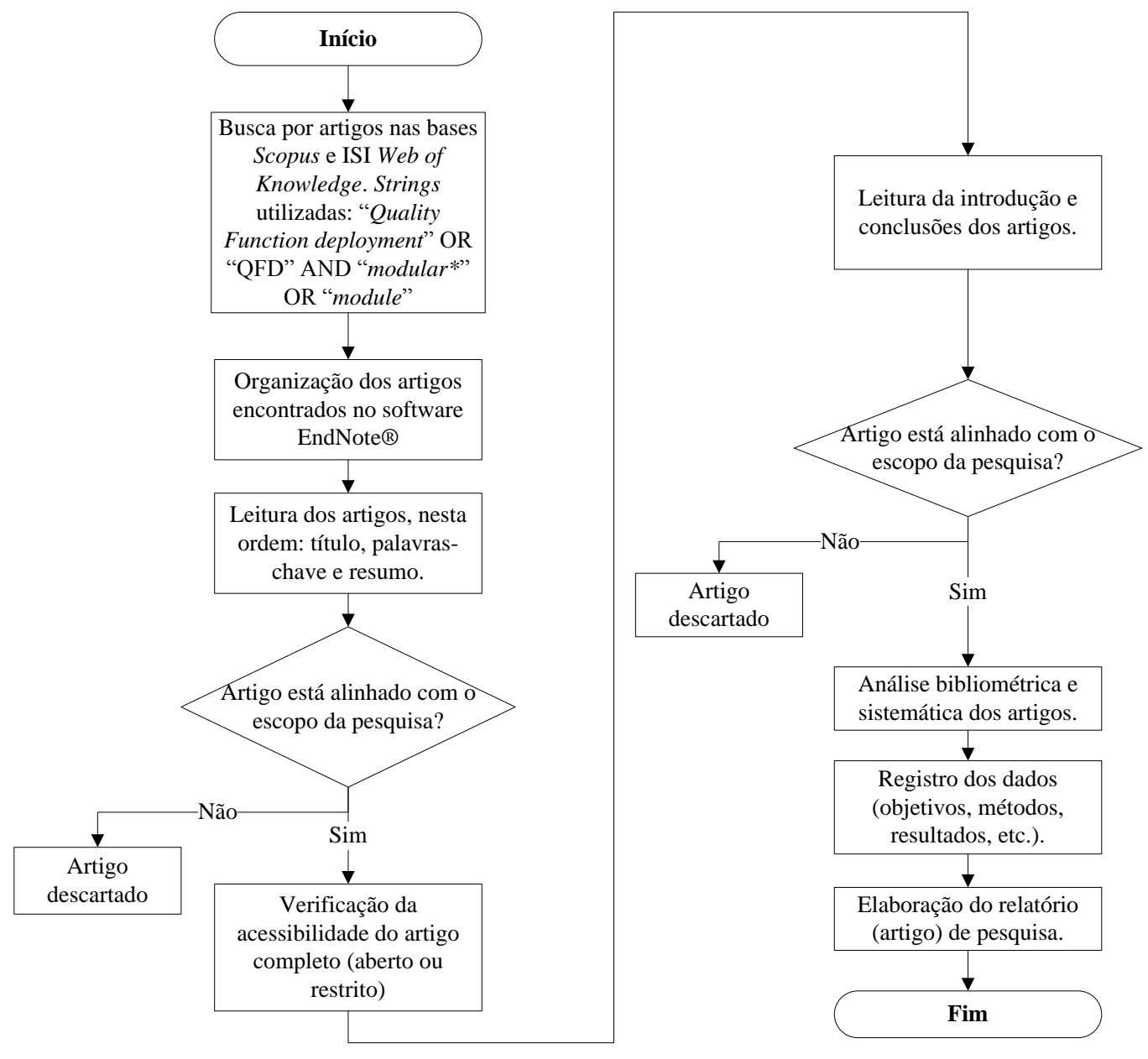

Fonte: Elaborado pelos autores (2012)

\section{Resultados e discussão}

Nesta seção, os resultados obtidos a partir da análise do portfólio bibliográfico considerado são apresentados, na seguinte ordem: organização e análise preliminar dos artigos; síntese (resumo) das publicações consideradas para análise. Por fim, a análise de conteúdo dos mesmos é mostrada, identificando os principais resultados quanto aos aspectos destacados nos procedimentos metodológicos.

\subsection{Organização e análise preliminar do portfólio de artigos}

Por meio das palavras chave citadas anteriormente, realizou-se as buscas nas bases de dados. Na Scopus, 49 artigos foram identificados, 24 na ISI Web of Knowledge 24 e 98 artigos na Compendex, totalizando-se 171 artigos no total. No entanto, duplicatas foram observadas dentro dessa quantidade de artigos, reduzindo o total para 85 artigos. Foram desconsiderados artigos publicados em periódicos chineses e japoneses com acesso restrito, restando 65 artigos. Em seguida, esses artigos foram lidos, preliminarmente, nesta sequência: título, palavras-chave e resumo 
(abstract). Assim, dos 65 artigos, 20 artigos foram considerados para uma análise de conteúdo mais aprofundada. Depois dessa filtragem, procedeu-se a leitura da introdução e conclusões do artigo, seguidos da leitura dos procedimentos metodológicos e resultados encontrados. Depois dessa leitura, verificou-se que 14 artigos se encontram dentro do contexto definido para o trabalho. A Tabela 1 sintetiza os resultados a cada filtragem realizada na análise bibliográfica.

Tabela $1-$ Filtros da pesquisa

\begin{tabular}{cc}
\hline Sequência dos filtros da pesquisa & $\begin{array}{c}\text { Quantidade } \\
\text { de artigos }\end{array}$ \\
Artigos - Scopus & 49 \\
Artigos - ISI Web of Knowledge & 24 \\
Artigos - Compendex & 98 \\
Total de artigos (somatório das bases) & 171 \\
Filtro 1 (duplicatas) & 85 \\
Filtro 2 (periódico chinês/japonês) & 65 \\
Filtro 3 (abstract e escopo do artigo) & 20 \\
Final - Artigos identificados e analisados & 14 \\
\hline Fon
\end{tabular}

Fonte: Elaborado pelos autores

Em seguida, foi registrado o número de publicações ao ano. Dos 14 artigos analisados, percebeu-se que apenas a partir de 2004 os trabalhos englobando modularidade e QFD começaram a ser desenvolvidos e publicados, conforme ilustra a Figura 2. Verifica-se, ainda, que os anos de 2007, 2010 e 2012 se destacam com 3 publicações cada, evidenciando-se que a adoção dos dois conceitos de forma integrada é recente. Quanto à abordagem metodológica, verificou-se que a maioria dos artigos é teórico-empírico e de dados de natureza qualitativa, sendo 11 artigos de modelagem teórica (desenvolvimento de modelos para posterior aplicação prática) e 3 de estudo de caso único. Não foram encontradas evidências acerca de outros métodos de pesquisa empregados, tais como survey, pesquisa-ação, simulação, experimento, revisão de literatura ou teóricoconceitual.

Em seguida, fez-se o levantamento das palavras-chave mais utilizadas nos trabalhos encontrados. A partir de um variação total de 62 palavras-chave utilizadas (desconsiderando as repetições ocorridas, 74 considerando a repetição das mesmas), percebeu-se que a palavra-chave de maior destaque é "quality function deployment", com 10 ocorrências, de certa forma esperado considerando-se o escopo do trabalho. Outras palavras-chave com mais destaque foram "house of quality", "QFD", "axiomatic design" (com duas ocorrências), o que pode denotar em utilização restrita a matriz da qualidade em alguns trabalhos analisados (no caso da primeira palavra-chave mencionada). Percebe-se dessa forma que as palavras-chave relacionadas ao QFD foram utilizadas 14 vezes (19\%), enquanto que os termos relacionados à modularidade teve um total de 12 ocorrências (16\%). 
Com relação às abordagens em produtos (bens de consumo) ou serviços, observa-se, preliminarmente que ainda há predominância da abordagem em produtos, uma vez o uso da modularidade e QFD no setor de serviços é recente. As palavras-chave relacionadas a serviços totalizam $9 \%$ do total, enquanto que as referentes a produtos, totalizam-se $28 \%$. A Tabela 2 ilustra as palavras-chave relacionadas ao QFD, modularidade, serviço e projeto/produto, tais como suas ocorrências e distribuição pelo portfólio de artigos. Todas as palavras-chave encontradas no portfólio bibliográfico de artigos se encontram disponíveis no Apêndice 1.

A partir das palavras-chave, foi possível identificar aspectos emergentes oriundos dos grupos de palavras considerados. Quanto ao QFD, percebeu-se que há uma tendência em utilização centrada na primeira matriz da qualidade (house of quality). Sobre a modularidade, foi observado o surgimento de diversos aspectos, tais como: o desenvolvimento de família de produtos, o particionamento dos produtos em módulos no projeto de produto modular, método de produção por módulo, modularidade e modularização em serviços e, ainda, uma variação do QFD, o modular function deployment (desdobramento das funções modulares).

Quanto às abordagens em produto ou serviço, percebe-se, preliminarmente, que há uma predominância de palavras-chave relacionadas aos produtos como bens de consumo (exemplos: physical product, conceptual design, design alternatives, etc.). Além disso, emergem diversos termos relacionados ao projeto, tais como modular design, redesign method, product variant design e design. Tanto na perspectiva de produto como de serviços houve destaque para dois termos: product service e product-service system, denotando o surgimento da abordagem de sistemas produto-serviço no âmbito de estudos sobre modularidade e QFD. 
Tabela 2 - Proporção das palavras-chave utilizadas no portfólio bibliográfico

\begin{tabular}{|c|c|}
\hline $\begin{array}{c}\text { Quality Function Deployment (10) } \\
\text { QFD (2) } \\
\text { House of quality (1) } \\
\text { HoQ (1) } \\
\text { Total: } 14 \text { ocorrências (19\%) }\end{array}$ & $\begin{array}{l}\text { Palavras-chave - Modularidade } \\
\text { Modular (1) } \\
\text { Modular design (1) } \\
\text { Modular driver (1) } \\
\text { Modular function deployment (1) } \\
\text { Modular product design }(1) \\
\text { Modular product family (1) } \\
\text { Modularity (1) } \\
\text { Modularization }(1) \\
\text { Module partition }(1) \\
\text { Production method module }(1) \\
\text { Service modularity }(1) \\
\text { Service modularization }(1) \\
\text { Total: } 12 \text { ocorrências }(\mathbf{1 6 \%})\end{array}$ \\
\hline $\begin{array}{l}\text { Integrated service product (1) } \\
\text { Product service (1) } \\
\text { Product-service system (1) } \\
\text { Service design (1) } \\
\text { Service modularity (1) } \\
\text { Service modularization (1) } \\
\text { Service quality assurance (1) } \\
\text { Total: } 7 \text { ocorrências (9\%) }\end{array}$ & $\begin{array}{c}\text { Palavras-chave - Projeto/Produto } \\
\text { Axiomatic design (2) } \\
\text { Collaborative product development (1) } \\
\text { Conceptual design (1) } \\
\text { Design (1) } \\
\text { Design alternatives (1) } \\
\text { Integrated service product (1) } \\
\text { Modular design (1) } \\
\text { Modular product design (1) } \\
\text { Modular product family (1) } \\
\text { Physical product (1) } \\
\text { Product configuration (1) } \\
\text { Product decision tree (1) } \\
\text { Product planning (1) } \\
\text { Product service (1) } \\
\text { Product strategy (1) } \\
\text { Product variant design (1) } \\
\text { Product variety (1) } \\
\text { Product-service system (1) } \\
\text { Quantified design structure matrix (1) } \\
\text { Redesign method (1) } \\
\text { Total: 21 ocorrências (28\%) }\end{array}$ \\
\hline
\end{tabular}

Fonte: desenvolvido pelos autores com base nos dados coletados (2012). Entre parênteses: número de ocorrência das palavras-chave

Posteriormente, levantou-se também os periódicos onde os 14 artigos foram publicados, conforme ilustra a Tabela 3. Nota-se que há uma grande dispersão, uma vez que os 14 artigos se encontram em 13 periódicos diferentes, não havendo uma revista de referência no tema, o que é justificável considerando que os trabalhos ainda são relativamente recentes. Ainda em relação aos periódicos, foram classificados segundo os respectivos fatores de impacto, conforme dados do Scimago Journal \& Country Rank (SJR). 
Tabela 3 - Portfólio de artigos: referências e respectivos periódicose fator de impacto

\begin{tabular}{ccc}
\hline Referência & Periódico & Fator de Impacto \\
\hline Qin e Wei (2012) & Journal of Software & 1,589 \\
Li et al (2012) & Computers in Industry & 1,529 \\
Geum et al (2012) & Computers \& Industrial Engineering & 1,103 \\
Lin e Pekkarinen (2011) & Journal of Business \& Industrial Marketing & 0,855 \\
Lo et al (2010) & Expert Systems with Applications & 2,075 \\
Torres et al (2010) & Concurrent Engineering - Research and Applications & 0,457 \\
Ko e Kuo (2010) & Concurrent Engineering - Research and Applications & 0,457 \\
Spinella et al (2009) & Journal of Materials Engineering and Performance & 0,584 \\
Miller e Nelson (2008) & Journal of Medical Devices - Transactions of the ASME & 0,476 \\
Wang e Lin (2007) & International Journal of Flexible Manufacturing Systems & 0,250 \\
Kondoh et al (2007) & Journal of Advanced Mechanical Design, Systems and & 0,226 \\
Hung et al (2007) & Manufacturing & 2,109 \\
Takai e Ishii (2006) & Int. Journal of Advanced Manufacturing Technology & 0,500 \\
Kreng e Lee (2004) & Journal of Mechanical Design & 0,500
\end{tabular}

Fonte: Elaborado pelos autores com base nos dados coletados e SJR (2012)

Observa-se que a maioria dos periódicos tem classificação relevante e de alto impacto, o que denota em publicações de maior qualidade e significativas contribuições científicas às áreas de atuação. Na próxima subseção, é feita uma síntese analítica dos 14 artigos do portfólio bibliográfico.

\subsection{Síntese das publicações sobre modularidade/QFD}

A partir dos 14 artigos analisados, foi possível observar algumas oportunidades, lacunas e características relacionadas à aplicação da modularidade com o QFD. Inicialmente, uma síntesede cada uma das 14 publicações é apresentado nesta subseção, seguido por uma discusão sobre o conteúdo desses trabalhos.

Qin e Wei (2012), considerando os requisitos dos clientes e a necessidade de oferecer produtos customizados, desenvolveram um método que, por meio do QFD, transformou e classificou os requisitos de cliente em especificações de projeto, que posteriormente foram tratadas de modo a organizar essas informações ao longo dos módulos de uma família de produtos. A principal contribuição dos autores foi a elaboração de um modelo que evita diversas combinações inválidas de módulos ao longo da família de produtos, aumentando a eficiência na configuração dos produtos e evitando o uso indevido de recursos em combinações inviáveis de módulos e componentes.

Li et al (2012) desenvolveram uma metodologia visando um processo de projeto modular interativo para o desenvolvimento de serviços, utilizando como estudo de caso para avaliação do método o projeto de um transformador elétrico. Os autores perceberam que o modelo teórico 
proposto contribuiu no aumento da satisfação dos clientes nas configurações de módulos físicos e serviços, ou seja, o método tem versatilidade para atender tanto bens de consumo (tangível) como os serviços que o envolve (intangível). No entanto, consideram que ainda é necessário implantar a metodologia desenvolvida em outras fases do ciclo de vida do produto, uma vez que no caso analisado, foi utilizada apenas na fase de projeto.

Geum et al (2012) também propuseram um modelo teórico, utilizando-se da Matriz (ou casa) da Qualidade modificada para a modularização de serviços, aplicando-o nos serviços de um restaurante industrial. Dentro da aplicação, os autores relataram que a modularidade tem potencial para desempenhar uma função importante no setor de serviços em várias perspectivas, apesar de a modularização de serviços ter sido raramente abordada. No caso em específico, o conceito foi útil para classificar de forma mais precisa os requisitos importantes para a qualificação e categorização dos serviços realizados, além de identificar os processos que mais impactam na satisfação dos clientes. Assim, neste estudo, evidencia-se que a modularidade nesse contexto (serviços) ainda é um assunto pouco explorado, emergindo como oportunidade de trabalhos futuros.

Assim como no trabalho anteriormente relatado, Lin e Pekkarinen (2011) realizaram um modelo teórico voltado para serviços, no setor logística de terceira parte, baseado no QFD, por meio da integração da matriz da qualidade e da modularidade, com o objetivo de desenvolver serviços logísticos de alta qualidade e ampla variedade de serviços. Neste trabalho, ressalta-se que os autores não se restringiram apenas à primeira matriz do QFD, elaborando um modelo conceitual de três níveis que, nesta ordem: captaram as necessidades e desejos dos clientes da empresa unidade de análise; transformaram essas informações em especificações de serviço e, por fim; converteram-as em requisitos de elementos que compõem os processos de serviço.

Torres et al (2010) integraram ferramentas de projeto junto com a abordagem de captação de conhecimento em um sistema CAD (Computer-Aided Design), e dentro dessa integração, utilizaram-se do QFD para a captação dos requisitos necessário para o desenvolvimento de um sistema PLM/CAD (Product Lifecycle Management/CAD), o qual teve seus módulos avaliados em relação à capacidade de suportar o fluxo de informações a serem utilizadas na fase de projeto conceitual, visando um sistema que minimize a descontinuidade de informações tais como: necessidades dos clientes, características-chave, parâmetros de projeto e parâmetros geométricos de projeto.

Lo et al (2010) também propuseram um modelo, do QFD baseado em gráficos morfológicos, visando o suporte à variantes de projeto de produtos simples e também os tecnicamente mais maduros. Como aplicação do modelo, foi utilizado um caso real do desenvolvimento de um mouse de computador. Nos resultados, os autores relatam que houve rápida geração de conceitos inovadores, com simultânea manutenção da viabilidade de manufatura. No entanto, oportunidades 
ainda foram identificadas para a sequência do estudo, como por exemplo incorporar métodos mais sofisticados ao modelo teórico, visando uma obtenção de informações mais complexas e úteis no desenvolvimento de produtos.

Ko e Kuo (2010) apresentaram um método sistemático para desenvolver variedade de produtos simultaneamente, utilizando-se de uma empresa de equipamentos eletrônicos como objeto de análise. Juntamente com o planejamento de mercado, os autores aplicaram o QFD para obter os requisitos de variedade e o grau de importância dos mesmos ao longo dos componentes dos produtos. Neste estudo, o QFD foi utilizado, essencialmente, para obter as informações necessárias ao desenvolvimento dos módulos. Como recomendação, os autores relatam a adequação do modelo teórico em outros tipos de produtos, visando o aumento de sua robustez.

Spinella et al (2009) descrevem o projeto conceitual, a modelagem, a otimização, o projeto detalhado e os testes virtuais de uma liga com memória de forma (LFA) (shape memory alloy SMA) concebida para maximizar o torque e o curso angular, limitando o tamanho do equipamento e o consumo elétrico, simultaneamente. Com o QFD, os autores visaram garantir a qualidade do dispositivo, respeitando as necessidades dos clientes durante o ciclo de vida do produto, que teve uma construção modular, resultando em melhor desempenho em termos de rotação, torque e customização. Em trabalhos futuros, há a oportunidade de melhorar o conceito em relação aos concorrentes no mercado da empresa estudada.

Miller e Nelson (2008) aplicaram o QFD para determinar os critérios de projeto importantes para o desenvolvimento de um instrumento cirúrgico modular, o qual foi projetado dessa forma para atender a diversas funcionalidades. Os autores relatam as contribuições do QFD no sentido de hierarquizar os requisitos de cliente, bem como sua correlação com as especificações de projeto para a concepção do instrumento multifuncional por meio da modularidade no projeto. Futuramente, deve-se utilizar outras técnicas adicionais para o desenvolvimento de um projeto mais seguro, considerando, para isso, o método FMEA (Failure Mode and Effects Analysis), priorizando os módulos que possuem maior risco de falhas.

Wang e Lin (2007) adaptaram o QFD para elaborar um método de rastreamento de defeitos para os módulos de produção para a customização em massa. Os resultados evidenciam que houve benefícios quanto à identificação dos requisitos necessários para uma adequação da customização em massa às expectativas dos clientes.

Kondoh et al (2007) propuseram um método para reprojeto de sistemas de produção, considerando o projeto de produto, baseado no QFD. Neste estudo, que analisou o sistema de produção de uma sonda de contato, pode-se perceber que o conceito de modularidade na produção foi abordado, uma vez que os autores, por meio da matriz da qualidade, identificaram os requisitos que foram transformados em características de produto, a partir dos quais foi possível identificar os 
processos produtivos essenciais para obter as características necessárias para o atendimento dos requisitos de clientes. A partir deste trabalho, emerge a possibilidade de acompanhar a implantação dos processos e a avaliação das propostas após essa implantação.

Hung et al (2007) propõem um modelo para o desenvolvimento colaborativo de produtos (CPD) visando a fabricação de produtos modulares. Os autores relatam que o método está alinhado com a estratégia da organização, diferente de outros métodos tradicionais, e contribui no diferencial competitivo das empresas no desenvolvimento de produtos. Entretanto, percebeu-se que, assim como a maioria dos 14 artigos analisados, os estudos foram centrados apenas na primeira matriz da qualidade. Assim, surge a possibilidade de o modelo ser ampliado, com a adoção de maior quantidade de matrizes, conforme as especificações e requisitos adicionais a serem considerados.

Takai e Ishii (2006), assim como a maioria dos demais autores, desenvolveram um método considerando matrizes da qualidade para captar os requisitos de clientes para, em seguida, priorizar as especificações de projeto mais importantes, seguida do desdobramento dessas especificações para os componentes de um projeto de um sistema magnético multipolo, onde cada um dos módulos (componentes) teve suas especificações e requisitos particulares. Em complemento, o modelo dos autores também considera o custo por componentes e módulos, utilizando-se do método target costing analisando, em conjunto, a viabilidade de obtenção do produto, considerando os custos e o desempenho projetado dos conceitos elaborados.

Por fim, Kreng e Lee (2004), com o objetivo de explorar a seleção de direcionadores para a modularidade por meio da integração e correlação de requisitos e componentes, desenvolveram um método para o projeto e fabricação de produtos modulares, considerando os benefícios da modularidade e da customização em massa. A principal limitação observada é a adoção de estudo de caso único como método de pesquisa, ou seja, os resultados são mais detalhados, porém não têm elevado grau de generalização, emergindo a oportunidade de se aplicar casos múltiplos para maior validação dos resultados, bem como aumentar a robustez do método desenvolvido.

Após a síntese dos artigos, desenvolveu-se a análise de conteúdo do portfólio bibliográfico, conforme descrito na próxima subseção.

\subsection{Análise das publicações sobre modularidade/QFD}

Antes da discussão e análise das publicações em relação ao QFD/Modularidade, é importante ressaltar que a matriz da qualidade não é definida como o QFD completo, como já detalhado na fundamentação teórica (seção 2.2) deste trabalho. O método consiste em realizar o desdobramento da qualidade, transformando as necessidades dos clientes em especificações de projeto (PUGLIERI et al, 2011). 


\subsubsection{Aspectos gerais das aplicações}

Inicialmente, percebeu-se que alguns trabalhos consideram a necessidade de produtos e serviços personalizados, ou seja, com um maior nível de customização, em suas revisões de literatura, o que foi transferido, posteriormente, para o desenvolvimento dos estudos, impactando especialmente no projeto conceitual de produtos/serviços. Este aspecto contribui para uma ampliação na busca por novos segmentos de mercado, bem como posterior personalização de módulos e/ou família de produtos desenvolvidas. Nesse contexto, o tipo de modularidade que se sobressai é a de projeto, pois a maioria dos trabalhos visa a concepção do produto de modo que possa ser personalizado de forma mais econômica do que os produtos com arquitetura mais integral.

\subsubsection{Abordagem utilizada do QFD}

A maioria das publicações não utiliza o QFD em sua plenitude, ou seja, restringem-se ao uso da primeira matriz da qualidade para obter os requisitos e necessidades dos clientes, confirmandose, dessa forma, o que foi observado na análise das palavras-chave mais utilizadas nos artigos (ver seção 4.1). Outros métodos e ferramentas são utilizados posteriormente para o desenvolvimento da modularidade nos produtos, característica a qual pode ser observada na literatura. Por exemplo, Wang e Lin (2007) relatam que o exercício de QFD mais simples utiliza apenas o diagrama da Matriz da Qualidade, que visa obter os requisitos do cliente e transforma-os em requisitos técnicos. Os autores complementam afirmando que a matriz (ou casa) da qualidade é a ferramenta mais utilizada no QFD. No entanto, ressalta-se que essa não engloba o QFD como um todo, trata-se apenas da primeira matriz do modelo conceitual do método.

No entanto, exceções podem ser encontradas, como é o caso do estudo de Lin e Pekkarinen (2011), onde os autores utilizaram um modelo conceitual de três níveis para o projeto de serviços de logística, para transformar os requisitos de cliente em especificações de serviço (projeto), que posteriormente foram convertidos em requisitos de elementos do processo. Há também os trabalhos de Hung et al (2007), que desenvolveu um modelo conceitual envolvendo a definição das necessidades (matriz tradicional do QFD), projeto de produto, projeto dos processos produtivos e, por fim, o planejamento da produção, e Takai e Ishii (2006), que desenvolveu método com diversos níveis considerando, adicionalmente, os custos de cada módulo de um sistema complexo por meio da técnica de target costing (custeio-alvo), visando analisar a viabilidade de obtenção dos conceitos elaborados.

\subsubsection{Análise dos métodos de pesquisa adotados}

A maior parcela dos estudos desenvolveram modelagem teórica, ou seja, desenvolveram modelos para desenvolvimento de produtos, processos ou serviços com base na literatura e suas lacunas, onde dentro dos modelos, o QFD foi considerado em determinadas etapas dos mesmos. Em 
sua maioria, o QFD é utilizado como a conexão entre os clientes e as organizações em nível interno, sempre se utilizando da casa da qualidade, correlacionando os requisitos de cliente com as especificações de projeto de produtos, geralmente nas etapas iniciais dos modelos. Ao passo que a modularidade é explicitada na concepção dos produtos/serviços após a definição das especificações de projeto.

\subsection{4. Áreas e setores de estudo dos artigos}

Também foi possível verificar os setores e áreas de estudo dos artigos analisados, bem como seus objetos de estudo. Nesse aspecto, percebe-se uma diversidade de aplicações: família (genérica) de produtos (QIN; WEI, 2012), transformador elétrico (LI et al, 2012), restaurante (GEUM et al, 2012), empresa de logística (LIN; PEKKARINEN, 2011), sistemas CAD (TORRES et al, 2010), mouses para computadores (LO et al, 2010), equipamentos eletrônicos (KO; KUO, 2010), ligas com memória de forma (LFA) (shape memory alloys - SMA) (SPINELLA et al, 2009), instrumentos cirúrgicos de laparoscopia (MILLER; NELSON, 2008), fabricantes de ferramentas manuais (exemplo: chaves de fenda) (WANG; LIN, 2007), sistema de produção de sondas de contato (KONDOH et al, 2007), sistemas inseridos em chips (HUNG et al, 2007), sistemas magnéticos (TAKAI; ISHII, 2006) e aspiradores de pó (KRENG; LEE, 2004). Isso ilustra que ainda não se pode, ainda, extrair uma área específica de destaque no tema QFD/Modularidade.

Ainda em relação aos setores de aplicação dos métodos elaborados, predominantemente, os estudos abordaram as áreas de computação e engenharia, ou seja, produtos e/ou serviços relacionados a esses ramos. Por fim, há uma tendência crescente da abordagem no setor de serviços, evidenciado nas publicações mais recentes como Lin e Pekkarinen (2011), Li et al (2012) e Geum et al (2012), embora a maior parte dos trabalhos ainda aborde o desenvolvimento de produtos como bens de consumo (conforme mencionado no parágrafo anterior). Os autores mencionados, de modo geral, desenvolveram modelos envolvendo os dois conceitos (QFD e modularidade) com o objetivo de desdobrar os requisitos dos clientes em especificações técnicas para projetar os serviços, utilizando-se dos conceitos de modularidade buscando a decomposição das etapas e processos que compõem o negócio das unidades de análise estudadas.

\subsubsection{Outros métodos e ferramentas utilizadas}

Especificamente nos trabalhos envolvendo modelagem teórica, percebeu-se o uso de outros métodos e ferramentas utilizadas para o desenvolvimento das aplicações: Failure Mode and Effects Analysis (FMEA), Axiomatic Design (AD - Projeto axiomático), Target costing (custeio-alvo), matriz de rastreamento de defeitos (DTM - Defects Tracking Matrix), lógicas de programação, ferramentas de CAD e simulação. Percebe-se que a maioria dessas técnicas e ferramentas envolvem o desenvolvimento e análise do projeto em uma perspectiva predominantemente interna, ou seja, em 
relação aos processos internos da organização. Além disso, a maioria dessas ferramentas é utilizada posteriormente à aplicação do QFD, com enfoque no desenvolvimento das especificações de projeto e definição do conceito do produto, levando em consideração, nesse processo, as necessidades e expectativas dos clientes (extraídas por meio da matriz da qualidade, principalmente).

Adicionalmente, percebeu-se, por meio da literatura, que há outros trabalhos que utilizam o projeto axiomático como forma de reduzir as dificuldades de aplicação do QFD pelas empresas no desenvolvimento de novos produtos (CAUCHICK MIGUEL et al, 2007), integração do QFD com o FMEA para análise dos conceitos desenvolvidos (FERNANDES; REBELATO, 2006), utilização do QFD para avaliação de sistemas CAD (ARAI; SHIMOMURA, 2005), entre outras aplicações. O que ratifica o potencial do QFD para a integração e complementação com outros métodos e técnicas da qualidade, tanto de forma integrada como sequencial no processo de desenvolvimento de produtos.

\subsubsection{Integração do QFD com os tipos de modularidade adotados}

Os trabalhos, predominantemente, abordam o QFD para desenvolver a modularidade no projeto de produto. Ou seja, os requisitos de cliente, depois de correlacionados com as especificações de projeto, são distribuídos no conceito do produto de forma que possam ser separados por módulos. Para isso, outras ferramentas também foram aplicadas para uma distribuição mais precisa, bem como melhor divisão dos módulos do produto em questão. As exceções são artigos como o trabalho de Kondoh et al (2007), que propuseram um método de reprojeto de sistemas de produção baseado no QFD, sendo assim uma abordagem na produção modular, além de considerar o projeto de produto. Outros artigos que não abordam o projeto de produto (no caso como bem de consumo) são os relacionados a serviços, como os já mencionados anteriormente (ver LIN; PEKKARINEN, 2011 e GEUM et al, 2012). Também foi percebido que a maioria das publicações trata da melhoria de produtos já existentes, ou seja, não aborda o desenvolvimento de produtos completamente novos, tratando, no máximo, de reprojetos/redesenhos ou inovações incrementais em produtos/serviços.

\subsubsection{Oportunidades emergentes do portfólio bibliográfico}

Quanto às oportunidades para investigações futuras relatadas no porfólio de artigos considerados (propostas pelos respectivos autores de cada estudo), essas foram inseridas no Quadro 1. Ressalta-se que apenas os artigos que apresentam, explicitamente, suas limitações e oportunidades, foram inseridos nesse quadro. 
Quadro 1 - Oportunidades para estudos futuros do portfólio de artigos

\begin{tabular}{|c|c|}
\hline Referência & Oportunidades para trabalhos futuros \\
\hline $\begin{array}{l}\text { Qin e Wei } \\
\text { (2012) }\end{array}$ & $\begin{array}{l}\text { O problema de como escolher os módulos exatos para os requisitos específicos de clientes ou } \\
\text { configurar produtos baseado na decomposição dos requisitos de cliente, ainda não foi resolvido; } \\
\text { Métodos para processar a linguagem online natural de clientes e como aplicar ferramentas de } \\
\text { tecnologia e comunicação virtual para obter os requisitos de clientes. }\end{array}$ \\
\hline Li et al (2012) & $\begin{array}{l}\text { Considerar a utilização do método proposto em outras fases do ciclo de vida do produto, uma vez } \\
\text { que, no caso estudado, foi utilizado na fase de projeto. }\end{array}$ \\
\hline $\begin{array}{l}\text { Geum et al } \\
(2012)\end{array}$ & $\begin{array}{l}\text { A modularização de serviços ainda é um tema pouco explorado, principalmente se comparado à } \\
\text { manufatura e projeto de produtos; } \\
\text { Aplicar o modelo teórico proposto pelos autores em um setor mais lucrativo que o de restaurantes } \\
\text { industriais; } \\
\text { Identificar os tipos de módulos ou modularidade que são especialmente apropriadas para a } \\
\text { aplicação em serviços, o que poderia gerar descobertas úteis para gerentes de empresas de serviços, } \\
\text { visando uma efetiva modularização dos mesmos; } \\
\text { Utilizar a abordagem proposta para aplicar a modularidade no desenvolvimento de novos serviços } \\
\text { (NSD - new service development). O que pode ser promissor, uma vez que a principal vantagem da } \\
\text { modularidade é fornecer variedade por meio da combinação criativa de módulos funcionais. }\end{array}$ \\
\hline $\begin{array}{l}\text { Lin e } \\
\text { Pekkarinen } \\
\text { (2011) }\end{array}$ & $\begin{array}{l}\text { Extensão do modelo teórico para outros negócios no setor de serviços; } \\
\text { Limitação: o trabalho conduziu um estudo de caso único em uma empresa de logística de terceira } \\
\text { parte (3PL - Third-Party Logistics) e suas empresas cliente (três empresas). Sugere-se assim a } \\
\text { adoção de mais estudos de caso para aumentar a robustez do modelo; } \\
\text { Focar o modelo nas interfaces entre módulos em diferentes níveis, em particular no contexto de } \\
\text { servir diversos clientes de diferentes indústrias sob a mesma plataforma de serviços. }\end{array}$ \\
\hline $\begin{array}{l}\text { Lo et al } \\
(2010)\end{array}$ & $\begin{array}{c}\text { Combinar os requisitos obtidos pelo modelo diretamente com os sistemas de gerenciamento de } \\
\text { dados de produto (PDM - Product Data Management); } \\
\text { Aplicação potencial: sistema PDM direcionado ao cliente, que rastreie a história do } \\
\text { desenvolvimento do produto relacionado aos requisitos de cliente; } \\
\text { Incorporação de métodos sofisticados como o Analytic Hierarchy Process (AHP) ao modelo } \\
\text { teórico proposto para obter informações mais abrangentes e, possivelmente, mais precisas das } \\
\text { necessidades dos consumidores. }\end{array}$ \\
\hline $\begin{array}{l}\text { Ko e Kuo } \\
(2010)\end{array}$ & $\begin{array}{c}\text { Abordagem proposta pode ser integrada a um sistema de planejamento de projeto para incrementar } \\
\text { o projeto colaborativo e desenvolvimento; } \\
\text { Aplicação do modelo em outros produtos pode ser importante para diversificar os casos e } \\
\text { enriquecer o modelo teórico. }\end{array}$ \\
\hline $\begin{array}{l}\text { Spinella et al } \\
(2009)\end{array}$ & Melhorias no desempenho da arquitetura desenvolvida, em relação aos concorrentes tradicionais. \\
\hline $\begin{array}{c}\text { Miller e } \\
\text { Nelson (2008) }\end{array}$ & $\begin{array}{l}\text { Posterior análise dos possíveis modos de falha do equipamento, e posterior redesenho dos } \\
\text { componentes (módulos) e mecanismos com maiores riscos. }\end{array}$ \\
\hline $\begin{array}{c}\text { Kondoh et } \\
\text { al(2007) }\end{array}$ & $\begin{array}{l}\text { Considerar operações de suporte à busca e rastreamento, posteriormente, no modelo desenvolvido. } \\
\text { Acompanhamento das modificações no sistema de produção após implantação. }\end{array}$ \\
\hline $\begin{array}{l}\text { Hung et al } \\
(2007)\end{array}$ & $\begin{array}{l}\text { Pesquisas recentes muito focadas na primeira matriz da qualidade. Considerar a utilização de mais } \\
\text { matrizes, ampliando o uso do QFD. }\end{array}$ \\
\hline $\begin{array}{l}\text { Takai e Ishii } \\
\quad(2006)\end{array}$ & $\begin{array}{l}\text { A seleção de conceito pode consistir de três etapas: triagem de conceitos inviáveis e priorização de } \\
\text { conceitos viáveis para análises mais detalhadas; obtenção de informações por meio da } \\
\text { prototipagem, e; seleção do conceito final incorporando uma análise de incertezas; } \\
\text { Desenvolvimento de um procedimento para a etapa de seleção de conceito final de produto, } \\
\text { retomando informações obtidas com o QFD/modularidade/target costing, deverá ser trabalhado em } \\
\text { estudos futuros. }\end{array}$ \\
\hline $\begin{array}{l}\text { Kreng e Lee } \\
\quad(2004)\end{array}$ & liação do modelo teórico, por meio da adoção de estudo de casos múltiplos. \\
\hline
\end{tabular}

Fonte: desenvolvido pelos autores

Observa-se que, essencialmente, há oportunidades em três direcionamentos: a continuação dos estudos desenvolvidos no setor e/ou produto em específico; a extensão dos modelos teóricos elaborados para aplicação em outros setores e; a adoção de estudos de casos múltiplos dos mesmos. Encerrando as discussões dos resultados, a Figura 2 ilustra, resumidamente, os principais resultados e oportunidades encontradas neste trabalho, seguidas das conclusões extraídas na seção final deste estudo. 
Figura 2 - Resumo dos principais resultados e oportunidades de pesquisa Principais resultados:
- Abordagem predominante em produtos como bens
de consumo;
- Tendência recente de trabalhos no setor de serviços;
- Maioria dos estudos limitada ao uso da $1^{\text {a }}$ matriz da
qualidade. Foco centrado no uso do QFD para obter
os requisitos de cliente;

Principais métodos de pesquisa adotados:

- Modelagem teórica (11 artigos - 79\%);

- Estudo de caso (3 artigos - 11\%);

- Inexistência na adoção de outros métodos como survey, simulação, experimento, pesquisa-ação, revisão de literatura e teórico-conceitual.

\section{Modularidade/QFD:}

Principais op ortunidades:

resumo dos resulta dos Setores de aplicação dos trabalhos:

- Utilização do QFD de forma plena (mais matrizes da qualidade - modelos conceituais);

- Familia (genérica) de produtos, transformador

- Ampliação dos modelos teóricos desenvolvidos por meio de estudo de casos múltiplos; elétrico, restaurante, empresa de logistica, sistemas CAD, mouses para computadores, equipamentos eletrônicos, ligas com memória de forma (LFA) (shape memory alloys - SMA), instrumentos - Definição precisa dos módulos com direcionamento especifico para os requisitos de clientes; cirúrgicos de laparoscopia, fabricantes de ferramentas manuais, sistema de produção de sondas de contato,

- Abordagem da modularidade/QFD no setor de serviços, área pouco exploradanesse contexto.

sistemas inseridos em chips, sistemas magnéticos e aspiradores de $p$

Fonte: desenvolvido pelos autores (2012)

\section{Conclusões}

Este trabalho teve como objetivo identificar e analisar estudos acerca dos temas modularidade e QFD (integrados), visando investigar as características referentes aos principais resultados quanto aos tipos de abordagem, métodos de pesquisa adotados, níveis de aplicação do QFD, setores de maior utilização dos conceitos integrados, tipos de modularidade e métodos e técnicas adicionais utilizadas desses trabalhos, além da identificação de perspectivas e principais oportunidades de pesquisas futuras no assunto. A partir disso, um levantamento das publicações existentes foi realizada, sendo possível extrair conclusões quanto às propostas dos trabalhos relacionados. Além disso, pode-se identificar lacunas inexploradas, as quais foram posteriormente descritas, com o intuito de apresentar os possíveis direcionamentos para trabalhos futuros envolvendo QFD e modularidade. Nesse aspecto, uma das contribuições do presente estudo é quanto ao método de pesquisa (teórico-conceitual e hipotético-dedutivo), uma vez que os artigos encontrados se utilizam apenas de modelagem teórica ou estudo de caso único.

Foi constatado que a maioria dos trabalhos tem preferência em utilizar apenas a primeira (e clássica) matriz da qualidade, possivelmente pelo fato de o principal interesse ser a captação dos interesses e expectativas dos clientes para posterior transformação em especificações técnicas de projeto. No entanto, ressalta-se que o QFD não se restringe apenas à essa matriz, podendo haver desdobramentos subsequentes, por exemplo, de tecnologia, confiabilidade, custos, processos produtivos, componentes, etc. Isso denota a falta da aplicação plena do QFD, ou seja, das outras matrizes da qualidade e modelos conceituais mais aprofundados para a obtenção dos produtos. 
Nesse sentido, nota-se a principal limitação nos estudos analisados: a não utilização do QFD de forma aprofundada, sendo poucas as exceções no portfólio bibliográfico considerado.

Pode-se concluir também que, de modo geral, as abordagens do QFD e da modularidade são realizadas de forma sequencial, não existindo uma integração mais efetiva desses conceitos. O QFD é abordado para captar as necessidades e expectativas dos clientes e, posteriormente, a modularidade é desenvolvida por meio de outras ferramentas e métodos, caracterizando assim essa independência entre os conceitos. A maior proximidade abordada entre os conceitos, considerando o portfólio bibliográfico, foi a transferência dos requisitos de cliente ao longo dos módulos, componentes e/ou subsistemas dos produtos/serviços objetos de estudo.

Observa-se também uma diversidade de aplicações dos estudos analisados. Desse modo, conclui-se que não há um setor ou área que se destaque no uso combinado da modularidade com o QFD, o que pode denotar em uma oportunidade de aplicações amplas para trabalhos futuramente, tanto em produtos como bens de consumo como em organizações prestadoras de serviços, considerando a tendência crescente dessa última abordagem no âmbito modularidade/QFD. Assim, evidencia-se essa possibilidade (investigações futuras no setor de serviços), uma vez que essa é uma tendência recente e a literatura ainda é imatura acerca das publicações envolvendo QFD e modularidade dentro dessa área.

Quanto aos tipos de modularidade adotados, percebeu-se que a modularidade em projeto é a que se sobressai perante às outras abordagens, pelo fato de os clientes e consumidores desejarem produtos mais customizáveis e personalizados, ao mesmo tempo que as empresas buscam a economia de escala. Em seguida, a modularidade em serviços também se destaca nos trabalhos mais recentes, apresentando-se como uma proposta de sistematizar os serviços considerando as funções dos mesmos, bem como um gerenciamento menos complexo e mais efetivo. Ainda, conclui-se que há uma predominância do desenvolvimento de modelos teóricos com posterior aplicação por meio de estudo de caso único dos mesmos. Assim, emerge também a possibilidade de ampliação dos trabalhos com estudos de casos múltiplos, envolvendo diversos setores para contínuas contribuições aos modelos, gerando maior robustez e versatilidade conforme a abordagem, bem como maior possibilidade de generalização dos dados e informações obtidos.

\footnotetext{
Abstract

Organizations increasingly receive pressures to produce goods and services of higher quality and customers and consumers suitability. Along with this, pressure for product development time reduction and product variety augment are also crescent intense. Thus, this paper identified and analyzed publications regarding modularity and quality function deployment (QFD), which are concepts widely used in recent years and considered important in the search towards higher quality and diversification. We performed a theoretical-conceptual research through a structured search and literature review regarding the topic. Results show gaps and opportunities for future research, as
} 
well as methodological aspects and results obtained by the analyzed papers. Hence, this study shows especially that most of the articles only explores the first quality function deployment matrix and adopts theoretical framework as research method. Besides there is a recent trend concerning the integrated adoption of the two concepts in the service sector.

Keywords: quality function deployment; QFD; modularity; theoretical-conceptual analysis.

\section{Referências}

AKAO, Y. QFD: Integrating customer requirements into product design. Cambridge: Productivity Press. 1990.

AKAO, Y. Introdução ao desdobramento da qualidade. Belo Horizonte: Fundação Christiano Ottoni. 1996.

ARAI, T.; SHIMOMURA, Y. Service CAD System - Evaluation and Quantification. CIRP Annals - Manufacturing Technology, v. 54, n. 1, p. 463-466, 2005.

ARNHEITER, E.D.; HARREN, H. Quality management in a modular world. The TQM Magazine, v. 18, n. 1, p. 8796, 2006.

cross ref

ASAN, U.; POLAT, S.; SERDAR, S. An integrated method for designing modular products. Journal of Manufacturing Technology Management, v. 15, n. 1, p. 29-49, 2004.

\section{cross ref}

BALDWIN, C.Y.; CLARK, K.B. Managing in an Age of Modularity. Harvard Business Review, v. 75, n. 5, p. 84-93, 1997.

BALDWIN, C.Y.; CLARK, K.B. Design Rules: The Power of Modularity. Cambridge: MIT Press. 2000.

BASK, A.; LIPPONEN, M.; RAJAHONKA, M.; TINNILÄ, M. Modularity in logistics services: A business model and process review. International Journal of Services and Operations Management, v. 10, n. 4, p. 379-399, 2011.

cross ref

BERTO, R.M.V.S.; NAKANO, D.N. A produção científica nos anais do Encontro Nacional de Engenharia de Produção: um levantamento de métodos e tipos de pesquisa. Produção, v. 9, n. 2, p. 65-76, 2000.

cross ref

CAMPAGNOLO, D.; CAMUFFO, A. What really drives the adoption of modular organizational forms? An institutional perspective from Italian industry-level data. Industry and Innovation, v. 16, n. 3, p. 291-314, 2009.

cross ref

CARDOSO, M.A.; KISTMANN, V.B. Modularização e design na indústria automotiva: o caso do modelo Fox da Volkswagen do Brasil. Revista Produção Online, v. 8, n. 4, 2008.

CARIDI, M.; PERO, M.; SIANESI, A. Linking product modularity and innovativeness to supply chain management in the Italian furniture industry. International Journal of Production Economics, v. 136, n. 1, p. 207-217, 2012.

\section{cross ${ }^{\text {ref }}$}

CARNEVALLI, J.A.; CAUCHICK MIGUEL, P.A. Empresas de referência na utilização do desdobramento da função qualidade. Produto \& Produção, v. 10, n. 1, p. 01-18, 2009.

CARNEVALLI, J.A.; CAUCHICK MIGUEL, P.A.; CALARGE, F.A. Requisitos de implantação do QFD e recomendações para reduzir dificuldades de uso: análise da literatura utilizando o método AHP. Revista Gestão Industrial, v. 1, n. 2, p. 18-32, 2005. 
CARNEVALLI, J.A.; VARANDAS JÚNIOR, A.; CAUCHICK MIGUEL, P.A. Uma Investigação sobre os Benefícios e Dificuldades na Adoção da Modularidade em uma Montadora de Automóveis. Produto \& Produção, v. 12, n. 1, p. 60-90, 2011.

CAUCHICK MIGUEL, P.A. Metodologia de Pesquisa em Engenharia de Produção e Gestão de Operações. Rio de Janeiro: Elsevier. 2010.

CAUCHICK MIGUEL, P.A.; CARNEVALLI, J.A.; CALARGE, F.A. Using Axiomatic Design for minimizing QFD application difficulties in NPD: research proposal and preliminary definition of first and second hierarchical levels. Product: Management \& Development, v. 5, n. 2, p. 127-132, 2007.

CHENG, L.C. Assessing performance of utilizing organizational modularity to manage supply chains: Evidence in the US manufacturing sector. International Journal of Production Economics, v. 131, n. 2, p. 736-746, 2011.

cross ref

CHRISTENSEN, T.B. Modularised eco-innovation in the auto industry. Journal of Cleaner Production, v. 19, n. 2-3, p. 212-220, 2011.

cross $r$ ref

CLAUSING, D. Total quality development: a step-by-step guide to world class concurrent engineering. Kansas City: American Society of Mechanical Engineers. 1994.

DEVADASAN. S.R.; KATHIVARAN, N.; THIRUNAVUKKARASU, V. Theory and practice of total quality function deployment: A perspective from a traditional pump-manufacturing environment. The TQM Magazine, v. 18, n. 2, p. 143-161, 2006.

cross ref

FERNANDES, J.M.R.; REBELATO, M.G. Proposta de um método para integração entre QFD e FMEA. Gestão \& Produção, v. 13, n. 2, p. 245-259, 2006.

cross ref

FILIPPINI, R. Operations management research: some reflections on evolution, models and empirical studies in OM. International Journal of Operations and Production Management, v. 17, n. 7, p. 655-670, 1997.

cross ref

FORMAGGIO, I.A.; CAUCHICK MIGUEL, P.A. Múltiplo Estudo de Casos sobre a Inserção do QFD no Processo de Desenvolvimento de Novos Produtos. Produto \& Produção, v. 10, n. 2, p. 62-86, 2009.

GERSHENSON, J.K.; PRASAD, G.J.; ZHANG, Y. Product modularity: Definitions and benefits. Journal of Engineering Design, v. 14, n. 3, p. 295-313, 2003.

cross ref

GEUM, Y.; KWAK, R.; PARK, Y. Modularizing services: A modified HoQ approach. Computers \& Industrial Engineering, v. 62, n. 2, p. 579-590, 2012.

cross ref

GRAZIADIO, T. Estudo Comparativo entre os Fornecedores de Componentes Automotivos de Plantas Convencionais e Modulares. Tese (Doutorado em Engenharia de Produção) - Universidade de São Paulo, São Paulo. 2004.

HUANG, C.C.; LIANG, W.Y.; CHUANG, H.F.; CHANG, Z.Y. A novel approach to product modularity and product disassembly with the consideration of 3R-abilities. Computers \& Industrial Engineering, v. 62, n. 1, p. 96-107, 2012. cross ref

HUNG, H.F.; KAO, H.P.; KU, K.C. Evaluation of design alternatives in collaborative development and production of modular products. International Journal of Advanced Manufacturing Technology, v. 33, n. 11-12, p. 1065-1076, 2007. 
KO, Y.T.; KUO, P.H. Modeling Concurrent Design Method for Product Variety. Concurrent Engineering: Research and Applications, v. 18, n. 3, p. 207-217, 2010.

KONDOH, S.; UMEDA, Y.; TOGAWA, H. Development of redesign method of production system based on QFD. Journal of Advanced Mechanical Design Systems and Manufacturing, v. 1, n. 1, p. 181-192, 2007.

cross ref

KRENG, V.B.; LEE, T.P. QFD-based modular product design with linear integer programming - A case study. Journal of Engineering Design, v. 15, n. 3, p. 261-284, 2004.

cross ref

KUSIAK, A.; HUANG, C.C. Development of modular products. IEEE Components, Packaging \& Manufacturing Technology, v. 19, n. 4, p. 523-538, 1996.

cross ref

LAGER, T. The industrial usability of quality function deployment: a literature review and synthesis on a meta-level.

R\&D Management, v. 35, n. 4, p. 409-426, 2005.

cross ref

LI, H.; JI, Y.J.; CU, X.J.; QI, G.N.; TANG, R.Z. Module partition process model and method of integrated service product. Computers in Industry, v. 63, n. 4, p. 298-308, 2012.

cross ref

LIMA, B.P.; MUNIZ JUNIOR, J.; FORTI, A.W. Projeto de embalagens para peças de automotivas aplicando o desdobramento da função qualidade (QFD). Revista Gestão Industrial, v. 8, n. 3, p. 126-141, 2012.

cross ref

LIN, Y.; PEKKARINEN, S. QFD-based modular logistics service design. Journal of Business \& Industrial Marketing, v. 26, n. 5, p. 344-356, 2011.

cross ref

LO, C.H.; TSENG, K.C.; CHU, C.H. One-Step QFD based 3D morphological charts for concept generation of product variant design. Expert Systems with Applications, v. 37, n. 11, p. 7351-7363, 2010.

cross ref

MILLER, D.J.; NELSON, C.A. Novel Mechanical Actuation of a Modular Laparoscopic Surgical Tool. Journal of Medical Devices-Transactions of the ASME, v. 2, n. 3, 2008.

MORRIS, D.; DONELLY, T.; DONELLY, T. Insights from Industry: Supplier Parks in the Automotive Industry. Supply Chain Management: An International Journal, v. 9, n. 2, p. 129-133, 2004.

cross ref

NUNES, B.; BENNETT, D. The Contribution of Modularity to Green Operations Practices. Brazilian Journal of Operations \& Production Management, v. 5, n. 2, p. 93-108, 2008.

PAHL, G.; BEITZ, W. Engineering design - A systematic approach. Darmstadt, Germany: Springer-Verlag. 1996.

PEKKARINEN, S.; ULKUNIEMI, P. Modularity in developing business services by platform approach. International Journal of Logistics Management, v. 19, n. 1, p. 84-103, 2008.

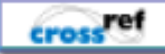

PELEGRINI, A.V. O processo de modularização em embalagens orientado para a customização em massa: uma contribuição para a gestão do design. Dissertação (Mestrado). Programa de Pós-graduação em Engenharia Mecânica, Universidade Federal do Paraná, 2004. 
PEROTTI, A.P.; SCHMIDT, A.S.; GODOY, L.P. QFD no planejamento da qualidade de capacetes para ocupantes de motocicletas. Revista Gestão Industrial, v. 5, n. 2, p. 134-158, 2009.

cross ref

PERSSON, M. Effects of changing a module's interface: a case study in an automotive company. International Journal of Automotive Technology and Management, v. 6, n. 3, p. 331-345, 2006.

cross ref

PINE, B.J. Mass customization: The new frontier in business. Boston: Harvard Business School Press. 1993.

PRIETO, E; CAUCHICK MIGUEL, P.A. Adoção da estratégia modular por empresas do setor automotivo e as implicações relativas à transferência de atividades no desenvolvimento de produto: um estudo de casos múltiplos. Gestão \& Produção, v. 18, n. 2, p. 425-442, 2011.

cross ref

PUGLIERI, F.N.; OMETTO, A.; CAUCHICK MIGUEL, P.A. Eco-design methods for developing new products based on QFD: a literature analysis. Product: Management \& Development, v. 9, n. 1, p. 23-29, 2011.

QIN, Y.; WEI, G. Product configuration flow from obtaining customer requirement to providing the final customized product. Journal of Software, v. 7, n. 2, p. 308-315, 2012.

SJR - SCIMAGO JOURNAL \& COUNTRY RANK. Disponível em: http://www.scimagojr.com/journalsearch.php. Acesso em: 15 jul. 2012.

SPINELLA, I.; MAMMANO, G.S.; DRAGONI, E. Conceptual Design and Simulation of a Compact Shape Memory Actuator for Rotary Motion. Journal of Materials Engineering and Performance, v. 18, n. 5-6, p. 638-648, 2009.

cross ref

STÄBLEIN, T.; HOLWEG, M.; MIEMCZYK, J. Theoretical versus actual product variety: how much customisation do customers really demand? International Journal of Operations \& Production Management, v. 31, n. 3, p. 350-370, 2011.

cross

SUNDBO, J. Modularization of service production and a thesis of convergence between service and manufacturing organizations. Scandinavian Journal of Management, v. 10, n. 3, p. 245-266, 1994.

TAKAI, S.; ISHII, K. Integrating target costing into perception-based concept evaluation of complex and large-scale systems using simultaneously decomposed QFD. Journal of Mechanical Design, v. 128, n. 6, p. 1186-1195, 2006.

\section{cross ref}

TERNINKO, J. The QFD, TRIZ and Taguchi connection: customer-driven robust innovation. Transactions from the Ninth Symposium on quality function deployment, v. 6, p. 441-445, Nov. 1997.

TORRES, V.H.; RÍOS, J.; VIZÁN, A.; PÉREZ, J.M. Integration of Design Tools and Knowledge Capture into a CAD System: A Case Study. Concurrent Engineering: Research and Applications, v. 18, n. 4, p. 311-324, 2010.

TSVETSKOVA, A.; GUSTAFSSON, M. Business models for industrial ecosystems: a modular approach. Journal of Cleaner Production, v. 29-30, p. 246-254, July 2012.

cross ref

WANG, C.S. Web-based modular interface geometries with constraints in assembly models. Computers \& Industrial Engineering, v. 56, n. 4, p. 1675-1686, 2009.

cross ref

WANG, H.; LIN, Z.Q. Defects tracking matrix for mass customization production based on House of Quality.

International Journal of Flexible Manufacturing Systems, v. 19, n. 4, p. 666-684, 2007. 


\begin{tabular}{|c|c|}
\hline attribute variable & module partition \\
\hline axiomatic design & morphological chart \\
\hline CAD & morphology analysis \\
\hline CATIA V5 & physical product \\
\hline collaborative product development & product configuration \\
\hline concept evaluation & product decision tree \\
\hline concept generation & product planning \\
\hline conceptual design & product service \\
\hline customer requirement classification & product strategy \\
\hline defects tracking matrix & product variant design \\
\hline design & product variety \\
\hline design alternatives & production method module \\
\hline FMEA & production system \\
\hline functional decomposition & product-service system \\
\hline HoQ & QFD \\
\hline house of quality & Quality function deployment \\
\hline integrated service product & quality value \\
\hline knowledge-based engineering & quantified design structure matrix \\
\hline laparoscopic surgical tool & redesign method \\
\hline linear integer programming & requirement and structure decomposition \\
\hline mapping & rotary actuator \\
\hline mass customization & service design \\
\hline minimally invasive surgery & service modularity \\
\hline modeling & service modularization \\
\hline modular design & service quality assurance \\
\hline modular driver & shape memory alloys \\
\hline modular function deployment & simulation \\
\hline modular product design & target costing \\
\hline modular product family & third party logistics \\
\hline modularity & third party vendors \\
\hline modularization & worth allocation \\
\hline
\end{tabular}

Fonte: Autores (2012)

\section{Dados dos autores:}

\section{Nome completo: Flávio Issao Kubota}

Filiação institucional: Universidade Federal de Santa Catarina (UFSC)

Departamento: Programa de Pós-graduação em Engenharia de Produção e Sistemas

Função ou cargo ocupado: Doutorando - bolsista CAPES

Endereço completo para correspondência (bairro, cidade, estado, país e CEP): Programa de Pósgraduação em Engenharia de Produção, Centro Tecnológico, UFSC, Campus Universitário

Trindade, Bairro Trindade, 88040-900 Florianópolis, Santa Catarina, Brasil

Telefones para contato: (48) 3721-2459

e-mail: flavioissao.kubota@gmail.com

\section{Nome completo: Paulo Augusto Cauchick Miguel}

Filiação institucional: Universidade Federal de Santa Catarina (UFSC) 
Departamento: Departamento de Engenharia de Produção e Sistemas (DEPS)

Função ou cargo ocupado: Professor Adjunto

Endereço completo para correspondência (bairro, cidade, estado, país e CEP): Departamento de Engenharia de Produção e Sistemas, Centro Tecnológico, UFSC, Campus Universitário Trindade, caixa postal 476, Bairro Trindade, 88040-900 Florianópolis - Santa Catarina - Brasil Telefones para contato: (48) 3721-7039

e-mail: cauchick@deps.ufsc.br

Submetido em: 05/12/2012

Aceito em: 08/08/2013 